\title{
Recent developments in the experimental elicitation of time preference*
}

\author{
Stephen L. Cheung ${ }^{\dagger}$ \\ The University of Sydney and IZA
}

First draft: 28 July 2015

This version: 23 November 2015

\begin{abstract}
This methodological survey reviews recent developments in the design of experiments to elicit individuals' time preferences, with a focus on the measurement or control for potentially non-linear utility. While the objective of a time preference experiment is usually to estimate parameters of a discount function, assumptions concerning the nature of utility may have an important influence upon these estimates. The survey classifies experiment designs on two dimensions: whether they assume an equivalence between utility under risk and over time, and whether they result in an estimate of the curvature of utility.
\end{abstract}

JEL classification: C91, D03, D90.

Keywords: time preference, discounted utility, instantaneous utility, choice list.

*I thank Peter Wakker for comments on an earlier draft, and the School of Economics and Finance at Queensland University of Technology for hospitality during the preparation of this paper.

†The University of Sydney, School of Economics, Merewether Building H04, Sydney NSW 2006, Australia (email: Stephen.Cheung@sydney.edu.au). 


\section{Introduction}

Many of the most important decisions that individuals make over their life course involve trade-offs over payoffs at different points in time. Controlled experiments, whether in a laboratory or field setting, provide a natural and attractive framework to study time discounting, as they enable researchers to systematically vary the amounts and dates of payoffs to infer information on decision-makers' preferences. Moreover, given the importance of time preference in applied settings, such experimental measures are increasingly included in studies where time preference is not the focal variable, but where instead the objective is to control for the effect of individual differences in future orientation in the study of other behaviors.

A theoretical challenge that has motivated significant recent advances in time preference research is the potential confounding influence of non-linear utility upon estimates of the discount function. This issue came to the wider attention of researchers through the survey of Frederick et al. (2002, pp. 381-2) although it was known to specialists prior to that time. Since then there has been considerable progress on controlling for utility in the measurement of time preference, yet little clear consensus over preferred methods has emerged. Indeed, a prominent strand of recent literature has been framed as a contest between two approaches in particular, namely the joint elicitation approach of Andersen et al. $(2008,2014)$ and the convex time budget approach of Andreoni and Sprenger (2012a).

This paper presents a methodological survey of recent experimental research on time preference, with a specific focus on issues of experimental design, in particular the measurement or control for potentially non-linear utility. Studies reviewed in the survey are classified on two dimensions: whether they assume an equivalence between utility under risk and over time, and whether they result in an estimate of the curvature of utility. The survey situates each of the leading approaches in a broader context by relating them to some less well-known designs. The aim of the survey is to expose the underlying assumptions and logic of each design that allow for utility and discounting to be disentangled, and the discount function identified. Because of this, some important issues are suppressed to the background. Firstly, I do not discuss econometric estimation in any detail. ${ }^{1}$ Secondly, I generally review aggregate estimates of the preferences of a representative agent. Finally, while the conceptual framework (set out in Section 2) is agnostic to the functional form of the discount function, I generally review estimates of annual exponential discount rates, as these are reported in most studies, readily interpretable, and comparable across studies. ${ }^{2}$

\footnotetext{
${ }^{1}$ In general, choice-based designs are amenable to maximum likelihood procedures such as those of Andersen et al. (2008, 2014) while matching and allocation designs are most commonly analyzed using some form of least squares - although a variety of alternative procedures have been proposed in the specific context of the convex time budget design (Andreoni and Sprenger, 2012a; Harrison et al., 2013; Andreoni et al., 2015). Some advantages and drawbacks of these broad approaches have been debated by Harrison et al. (2013) and Andreoni et al. (2015).

${ }^{2}$ Also, consistent with contemporary expectations in experimental economics, I focus on studies in which all choices were potentially for real payment. Given my focus on the logical structure of experiment design, this might appear to be an arbitrary choice. However, since the use of purely hypothetical incentives gives additional degrees of freedom to the researcher, it is not always clear how a hypothetical design may be transferred to a fully incentive-compatible setting.
} 


\section{Conceptual framework: Discounted utility}

I follow the vast majority of experimental literature in assuming that a stream of time-dated payoffs (to be delivered with certainty) is evaluated according to its discounted utility:

$$
D U=\sum_{t} D(t) \cdot v\left(x_{t}\right)
$$

where $D(\cdot)$ is a discount function with $D(0)=1$ and $D^{\prime}<0$, and $v(\cdot)$ is an instantaneous utility function with $v(0)=0, v^{\prime}>0$ and $v^{\prime \prime} \leq 0$. Letting the date of the experiment be $0, D(t)$ is the weight attached to utility $t$ periods in the future relative to utility today.

Instantaneous utility $v(\cdot)$ in choice over time is analogous to Bernoulli utility $u(\cdot)$ in choice under risk. For example, under expected utility a bundle of state-contingent payoffs is evaluated according to:

$$
E U=\sum_{s} p(s) \cdot u\left(x_{s}\right)
$$

where $p(s)$ is the probability of state $s$. Under expected utility, the concavity of Bernoulli utility captures a decision-maker's risk aversion (preference for more equal payoffs over different states of nature). Similarly under discounted utility, the concavity of instantaneous utility captures her attitude toward intertemporal substitution (preference to smooth payoffs over different points in time).

Although risk aversion and intertemporal substitution are in principle distinct preferences, Bernoulli and instantaneous utility are often assumed to be one and the same. This is usually explicit when risk and time interact - both in the standard model of discounted expected utility and some behavioral models (e.g., Halevy, 2008; Epper and Fehr-Duda, 2012). Perhaps because of this, the assumption is sometimes made even when risk and time are not inherently intertwined, such as when eliciting time preference under certainty. As will be seen, the limited experimental evidence on the equivalence of utility for risk and time is mixed at best. This has implications not only for experimental methodology, but also for the modeling of interactions between risk and time. In particular, the Epstein and Zin (1989) model of recursive preferences has proven useful in macroeconomics and finance because it allows for just such a separation.

While the full design of an experiment may involve multiple payment dates, in any given decision a subject typically makes a choice involving only two dates. ${ }^{3}$ In this case (1) reduces to:

$$
D U=D(t) \cdot v\left(x_{t}\right)+D(t+k) \cdot v\left(x_{t+k}\right)
$$

\footnotetext{
${ }^{3}$ As will be seen, depending upon the design the subject may receive payment on one or both of these dates.
} 
where $t$ is the "front-end delay" to the sooner date, and $k$ is the "back-end delay" between dates.

Equations (1) and (3) are agnostic with respect to the functional form of $D(t)$. Well-known specifications include the standard exponential model $D(t)=1 /(1+\rho)^{t}$ where $\rho$ is the discount rate, ${ }^{4}$ and the quasihyperbolic model $D(t)=\beta /(1+\rho)^{t}$ where $\beta$ captures present bias. A wide variety of other models have been proposed, and many of them are reviewed by Andersen et al. (2014, Section 2 and Appendix D). ${ }^{5}$

The primary objective of a time preference experiment is usually to estimate parameters of $D(t)$. However as (1) and (3) make clear, a subject's choices are guided by both the discount and utility functions. Therefore to make unbiased inferences about one, it is necessary to acknowledge the influence of the other. To see this, consider the absolute slope of an indifference curve of (3) in $\left(x_{t}, x_{t+k}\right)$ space:

$$
-\left.\frac{d x_{t+k}}{d x_{t}}\right|_{\overline{D U}}=\frac{D(t)}{D(t+k)} \cdot \frac{v^{\prime}\left(x_{t}\right)}{v^{\prime}\left(x_{t+k}\right)}
$$

This is a product of two terms: $D(t) / D(t+k)$ is the relative value of utility at $t$ compared to $t+k$ (an inverse discount factor), while $v^{\prime}\left(x_{t}\right) / v^{\prime}\left(x_{t+k}\right)$ captures the effect of diminishing marginal utility upon the payoffs. For the special case of linear utility, the second term drops out and a subject's choices are determined solely by the discount function. More generally, if utility is concave then as $x_{t+k}$ falls relative to $x_{t}$ the second term declines in value, generating conventional convex indifference curves that "bow in" toward the origin. Finally, notice that even when utility is not linear the second term cancels out at $x_{t+k}=x_{t}$, where the indifference curve intersects the diagonal and the subject receives equal payoffs on both dates.

\section{Base camp: The binary choice list}

To illustrate the effect of non-linear utility, consider the classic choice list design of Coller and Williams (1999) and Harrison et al. (2002). A subject makes a series of binary choices between alternatives that pay solely on either the sooner or later payment date. As the subject moves through the list, the smaller sooner option is typically fixed while the larger later one progressively improves, implying increasing interest rates for delay of payment. Assuming linear utility, the point at which a subject first switches to accepting delayed payment gives a measure of her (exponential) discount rate. ${ }^{6}$ This basic approach remains popular today.

Suppose that, on the basis of this design, a subject is found to be indifferent between $X$ at date $t$ (and nothing at $t+k$ ), or $Y>X$ at $t+k$ (and nothing at $t$ ). Then, one can infer that $D(t+k) / D(t)=v(X) / v(Y)$, a $k$-period discount factor for utility. The difficulty, of course, is that $v(\cdot)$ is unobserved. If one assumes that utility is linear, when in fact it is concave, one instead infers that $D(t+k) / D(t)=X / Y$, a $k$-period discount factor

\footnotetext{
${ }^{4} \delta \equiv 1 /(1+\rho)$ is the discount factor for one period of delay. The subject is more patient as $\rho$ is close to zero, or $\delta$ close to one.

${ }^{5}$ Analogously, a popular alternative to expected utility is to generalize (2) by replacing $p(s)$ with a non-linear probability weight, while retaining the additive structure; see Fehr-Duda and Epper (2012) for a review of the probability weighting function.

${ }^{6}$ In practice, as the larger later option increases in discrete increments, it is only possible to infer bounds on the discount rate.
} 
for money. Since concave utility implies that $v(x)$ increases less than proportionately with $x$, it follows that $X / Y<v(X) / v(Y)$. One thus underestimates the subject's discount factor, overestimating how impatient she is. Intuitively, both diminishing marginal utility and time discounting reduce the attractiveness of the larger later option. Therefore, if the former is assumed away then the effect of the latter is overstated. These observations have motivated a series of advances in the design of experiments to measure (or otherwise control for) utility in the elicitation of time preference, which are the focus of this survey.

\section{Assuming equivalence of utility for risk and time}

\subsection{Joint elicitation}

The most influential experiment design to measure time preference controlling for non-linear utility is the joint elicitation strategy of Andersen et al. (2008). This uses the binary choice approach to infer the discount function conditional upon utility, while a risk preference task is used to infer the utility function. The approach thus assumes equivalence of utility for risk and time, which is also the default assumption in standard theory. Moreover the measurement of utility is better understood in the context of risk than any other domain, ${ }^{7}$ and until quite recently little was known about how to infer utility outside of this domain.

The specific risk preference task used by Andersen et al. (2008) is based upon Holt and Laury (2002), a very well-known experiment that itself builds upon the logic of a choice list. In subsequent work, Andersen et al. (2014) further refine their approach, including by dispensing with the choice list format in favor of presenting choices one at a time; nonetheless, the underlying logic remains unchanged.

While joint elicitation assumes equivalence of utility for risk and time, a strength of the approach is that it need not assume expected utility under risk. This is notable because under alternatives such as rankdependent utility (Quiggin, 1982), risk aversion in a task such as Holt and Laury may be attributable to both the utility function and non-linear probability weighting. Since it is only the former that is applicable to the measurement of time preference, neglecting the possibility of probability weighting may cause estimates of utility to be misspecified, resulting in biased estimates of the discount function.

Andersen et al. (2008) conduct an experiment using a representative sample of 253 adult Danes, with parameters $t=1$ month, $k$ taking six values up to 24 months, $X=$ DKK 3,000, and annual interest rates up to $50 \%$. There was a $10 \%$ chance that each subject received real payment for one of her choices.

Using binary choice data for time while assuming linear utility, Andersen et al. estimate an annual discount rate of $25.2 \%$. They also find substantial aversion to risk, with an estimated CRRA coefficient of 0.74 under expected utility. ${ }^{8}$ The effect of correcting for this amount of utility curvature is to substantially lower the

\footnotetext{
${ }^{7}$ See Harrison and Rutström (2008) for a comprehensive survey.

${ }^{8}$ This is the parameter $\alpha$ in $u(x)=x^{1-\alpha} /(1-\alpha)$ where $\alpha=0$ represents linear utility, while $\alpha>0$ implies concave utility. Some papers instead estimate the power function $u(x)=x^{a}$ where $\alpha=1-a$. I report the CRRA parameter throughout.
} 
estimated discount rate to $10.1 \%$. Andersen et al. (2014, Section 5.3 and Appendix F) confirm that the main implications of joint elicitation are robust to alternatives to expected utility. While they indeed find evidence of non-linear probability weighting, most aversion to risk derives from the utility function. ${ }^{9}$

\subsection{Probability-based methods}

Although not as influential as joint elicitation, two other designs have been proposed to estimate discount rates, controlling for non-linear utility, while assuming equivalence of utility for risk and time. Both offer the tantalizing possibility that this may be done without explicitly estimating utility itself, and follow a similar logic of "translating" the back-end delay into an "equivalent" variation in the probability of payment. In each case, the price paid for bypassing utility is that it is necessary to assume expected utility under risk.

Laury et al. (2012) introduce a choice list design using a single nonzero payoff $M$. A subject makes a series of binary choices between a lottery that pays $M$ at date $t$ with probability $p_{t}$ (and zero otherwise), or one that pays the same amount at $t+k$ with probability $p_{t+k} \geq p_{t}$, with $p_{t+k}$ improving through the list. This design thus interacts risk with time. Assuming discounted expected utility, at a point of indifference one infers that:

$$
D(t) \cdot\left[p_{t} \cdot u(M)+\left(1-p_{t}\right) \cdot u(0)\right]=D(t+k) \cdot\left[p_{t+k} \cdot u(M)+\left(1-p_{t+k}\right) \cdot u(0)\right]
$$

Normalizing $u(0)=0$ this simplifies to:

$$
\frac{D(t+k)}{D(t)}=\frac{p_{t}}{p_{t+k}}
$$

Laury et al. conduct an experiment using 103 US student subjects, with parameters $t=3$ weeks, $k=9$ weeks, $M=\mathrm{USD} 200, p_{t}=0.5$ and $p_{t+k} \in[0.5,0.647]$ (implying annual interest rates up to $347 \%$ ). They also conduct a version of joint elicitation using the same subjects, to permit comparison between the methods.

In their replication of joint elicitation, Laury et al. (2012) estimate an annual discount rate of 55.5\% assuming linear utility. This falls to $14.1 \%$ after correcting for utility using a risk preference task. In their main probabilistic discounting task Laury et al. estimate a discount rate of $12.2 \%$, with a confidence interval that overlaps substantially with that obtained through joint elicitation but not with that assuming linear utility. ${ }^{10}$

A drawback of this design is that it requires subjects to discern very fine increments in $p_{t+k}$, as small as 0.001. As it turns out, one-third of subjects switch at the first non-zero interest rate, where $p_{t+k}$ increases

\footnotetext{
${ }^{9}$ Indeed, they estimate an S-shaped weighting function, as opposed to the inverse-S typically presumed. As a result, utility is actually more concave after allowing for probability weighting, and the estimated discount rate falls insignificantly.

${ }^{10}$ By combining their probabilistic discounting and joint elicitation data, Laury et al. estimate a specification that allows for probability weighting (as well as delivering an estimate of the utility function). However, by itself, their probabilistic discounting task can only elicit the discount rate without knowledge of utility under the assumption of expected utility.
} 
from 0.500 to 0.501 . These subjects might be very patient, but it could also be that the presentation makes increasing probability most salient, causing them to neglect the back-end delay (which is constant).

Instead of interacting risk with time, Takeuchi (2011) elicits separate choices under risk and over time using matched pairs of payoffs. On one hand, he elicits $p_{Y}$ such that a subject is indifferent between $X$ with a given probability $p_{X}$ (and zero otherwise), or $Y>X$ with probability $p_{Y}$. From this, and assuming expected utility, $u(X) / u(Y)=p_{Y} / p_{X}$. On the other hand, and using the same payoffs, he elicits $k$ such that the same subject is indifferent between $X$ at a given date $t$, or $Y$ at $t+k$. From this, $D(t+k) / D(t)=v(X) / v(Y)$. Finally, invoking equivalence of utility for risk and time $u(\cdot) \equiv v(\cdot)$, one infers that:

$$
\frac{D(t+k)}{D(t)}=\frac{p_{Y}}{p_{X}}
$$

Comparing this to (5) highlights the similarity in underlying logic of the Laury et al. (2012) and Takeuchi (2011) designs, the main difference being in the procedure used to elicit an equivalent probability: a single choice list in Laury et al. versus two matching tasks in Takeuchi.

Takeuchi (2011) conducts an experiment using 56 US student subjects, with parameters $p_{X}=1, t=0$, and ten combinations of $X$ and $Y$ taking values between USD 5 and 25. Since $D(0)=1$, (6) reduces to $D(k)=p_{Y}$, where both $k$ and $p_{Y}$ are elicited from the same subject in separate matching tasks. Each such pair thus identifies a single point on that subject's discount function.

Takeuchi fits a generalized Weibull discount function for each subject. For 33 subjects for whom this works well, the average discount function is hyperbolic. For these subjects, when linear utility is imposed (by substituting $X / Y$ instead of $p_{Y} / p_{X}$ in (6)), the discount rates increase, consistent with concave utility. ${ }^{11}$ However, for the remaining subjects the fit of the model is conspicuously poor, and is considerably improved when linear utility is instead imposed. For those subjects, this may be suggestive of violation of the assumption of equivalence of utility for risk and time.

\section{Inferring utility directly from choices over time}

The approaches considered to this point involve choices over alternatives in which a subject only receives payment on a single date. A key insight of the next set of designs is that to identify the curvature of instantaneous utility it is necessary to offer choices over alternatives that pay at both $t$ and $t+k$, and to vary the size of the difference between $x_{t+k}$ and $x_{t}$. In other words, to identify intertemporal substitution, subjects should have the opportunity to choose the extent to which they wish to smooth payments over time. ${ }^{12}$

\footnotetext{
${ }^{11}$ One unappealing feature of Takeuchi's results is that the estimated annual discount rate is extremely high at $726 \%$. This is striking given that it controls for non-linear utility, and uses a similar logic and subject pool to Laury et al. (2012). Contributing factors may include: the use of matching tasks in which subjects' responses are open-ended, the absence of any front-end delay, and the smaller magnitude of payoffs compared to Laury et al..

${ }^{12}$ As (4) makes clear, the impact of non-linear utility increases with the difference between $x_{t+k}$ and $x_{t}$, vanishing when $x_{t+k}=x_{t}$. If one of the payoffs is always zero, as in the classic binary choice design, this difference is effectively at a maximum. Analogously
} 


\subsection{The convex time budget design}

The convex time budget (CTB) design of Andreoni and Sprenger (2012a) is the leading alternative to the joint elicitation approach. Recall that the binary choice list design offers a series of choices between $X$ at date $t$ (and nothing at $t+k$ ) or $Y$ at $t+k$ (and nothing at $t$ ). Andreoni and Sprenger note that when utility is linear - as was assumed in the original applications of the binary choice design - restricting the choice set to these corners does not constrain subjects' optimal choices. However if utility is concave then, given the opportunity, subjects would prefer a convex combination to either extreme.

Andreoni and Sprenger (2012a) design an experiment in which subjects may choose any allocation they wish from the linear budget line connecting $(X, 0)$ and $(0, Y)$ :

$$
\frac{Y}{X} \cdot x_{t}+x_{t+k}=Y
$$

where the implied interest rate over $k$ periods is $r=\frac{Y}{X}-1$. If instantaneous utility $v(\cdot)$ is concave, discounted utility (3) is maximized at an interior solution where:

$$
\frac{D(t)}{D(t+k)} \cdot \frac{v^{\prime}\left(x_{t}\right)}{v^{\prime}\left(x_{t+k}\right)}=1+r
$$

As the interest rate varies, a subject's choices trace out a price expansion path in $\left(x_{t}, x_{t+k}\right)$ space. As (7) makes clear, these choices depend on both the utility and discount functions. Therefore to disentangle the two, the full design varies both the interest rate and payment dates orthogonally across CTB decisions.

Andreoni and Sprenger conduct an experiment using 97 US student subjects, with $t \in\{0,1,5\}$ weeks, $k \in$ $\{5,10,14\}$ weeks, $Y=\mathrm{USD} 20$ or $25,{ }^{13}$ and annual interest rates up to $1,300 \%$. Given these parameters, if the concavity of utility typically found in risk preference experiments were to also characterize intertemporal substitution, one would expect to observe a very strong preference for interior allocations, quite close to the midpoint of the budget set, notwithstanding the high interest rates on offer. ${ }^{14}$

Andreoni and Sprenger (2012a) instead find that most choices are corner solutions: overall, $17.1 \%$ are all-sooner and $52.4 \%$ all-later. They estimate an annual discount rate of $30.0 \%$ and find that utility is significantly concave, with a CRRA coefficient of 0.08 . However, this is much closer to linearity than the estimates typically obtained from risk preference data, as used in the joint elicitation approach.

Harrison et al. (2013) critique Andreoni and Sprenger's use of a non-linear least squares estimator, which seeks to explain the mean allocation, given that the mass of data is at the corners and not close to the

in Holt and Laury for risk, the curvature of Bernoulli utility is identified from the fact that the difference in payoffs between states (rolls of a ten-sided die) is small in lottery A, whereas it is large in lottery B. See Cheung (2015b) for related discussion.

${ }^{13}$ Whereas Andersen et al. (2008) vary interest rates by fixing $X$ and increasing $Y$, Andreoni and Sprenger fix $Y$ and decrease $X$.

${ }^{14}$ See Harrison et al. (2013, Table 1) for a detailed analysis. 
mean. Harrison et al. propose a multinomial logit estimator, which seeks to explain the entire distribution of choices. Applying this to the Andreoni and Sprenger (2012a) data they estimate significantly convex utility, with a CRRA coefficient of -0.36 for a representative agent - a result explained by the need for the estimator to account for choices at both corners. Nonetheless, this does not alter the observation that the incidence of corner solutions is incompatible with the concavity of utility found in risk preference experiments.

\subsection{The convex time budget under risk}

Andreoni and Sprenger (2012b) conduct a second experiment in which they extend the CTB approach to settings in which payments are subject to risk. Their main result is that when payments on both dates are sent with probability 0.5 , the incidence of corner solutions falls to $26.1 \%$ compared to $80.7 \%$ when both payments are certain. One interpretation, hinted at by their title "Risk preferences are not time preferences", is that utility under risk may be more concave than under certainty.

The difficulty with this explanation is that because the experiment involves an interaction between risk and time, the door is opened for a third preference, known as correlation aversion (Richard, 1975; Epstein and Tanny, 1980), to explain the result. Recall that risk aversion is characterized under expected utility by concavity of Bernoulli utility, and relates to the preference for more equal payoffs across states of nature, while intertemporal substitution is characterized by concavity of instantaneous utility, and relates to the preference to smooth payoffs over time. Correlation aversion is characterized by concavity of intertemporal utility, and gives rise to a preference to spread risks more equally over time. ${ }^{15}$

In particular, since Andreoni and Sprenger (2012b) realize payments at $t$ and $t+k$ using two independent lotteries, a subject can diversify these risks by choosing an interior solution whereas at a corner solution all payment hinges on a single lottery. Since this diversification motive does not operate when all payments are certain, it provides an alternative explanation for their result. ${ }^{16}$ Cheung (2015a) demonstrates how, even assuming equivalence of Bernoulli and instantaneous utility, the result may be explained by correlation aversion. He reports two replication experiments in which diversification is not possible; in one the result disappears completely, while in the other it is substantially diminished. Finally, Cheung estimates a model that allows for non-linear intertemporal utility, and confirms the correlation aversion hypothesis.

Epper and Fehr-Duda (2015) and Schmidt (2014) offer alternative explanations for Andreoni and Sprenger's result. Epper and Fehr-Duda invoke rank-dependent probability weighting, while Schmidt considers saving and borrowing outside the lab. In each case, the arguments turn on the use of independent lotteries to realize payment risk, and do not require separation between utility for risk and time. They thus reiterate that Andreoni and Sprenger do not establish conclusively that "Risk preferences are not time preferences".

\footnotetext{
${ }^{15}$ That is, the additive expression in (3) is replaced by a concave transformation. Andersen et al. (2011) build upon joint elicitation to design an experiment to identify correlation aversion, and find that their subjects are indeed correlation averse. Ebert and van de Kuilen (2015) also document correlation aversion in choice over time (as well as in other domains).

${ }^{16}$ This concern does not arise for Laury et al. (2012), who also interact risk with time, because their binary choice design ensures that a subject only ever faces the realization of a single risk.
} 
Thus to summarize the discussion of the CTB method: while the prevalence of corner solutions in Andreoni and Sprenger (2012a) is incompatible with the concavity of utility elicited under risk, the results of Andreoni and Sprenger (2012b) do not provide clear evidence for the separation of risk preference from intertemporal substitution due to the possibility of intertemporal diversification in their experiment design.

\subsection{Comparing utility for risk and time}

To avoid the confound encountered by Andreoni and Sprenger (2012b), it is necessary to compare the curvature of Bernoulli utility elicited under risk to instantaneous utility elicited over time, without interacting the two domains. Two recent studies have sought to do exactly this. Moreover, both studies do so using binary choices that are no more complex than those used in risk preference experiments such as Holt and Laury (2002), which find concave utility under risk. This is notable because it avoids the possibility that the finding of near-linear utility over time in Andreoni and Sprenger (2012a) - driven by a prevalence of corner solutions - might be an artifact of the greater complexity of the CTB task compared to binary choices.

Abdellaoui et al. (2013) use sequences of binary choices to elicit the (certain, immediate) payoff that makes a subject indifferent to either a risky or temporal bundle of payoffs. ${ }^{17}$ Thus for risk, they elicit the certainty equivalent $X_{c}$ of a gamble that pays $X_{g}$ with probability $p$, or $X_{b}<X_{g}$ otherwise:

$$
u\left(X_{c}\right)=w(p) \cdot u\left(X_{g}\right)+[1-w(p)] \cdot u\left(X_{b}\right)
$$

where $w(p)$ is a rank-dependent probability weight. The payoffs $\left(X_{g}, X_{b}\right)$ vary across decisions, but $p$ is fixed so it is only necessary to identify a single probability weight. For time, Abdellaoui et al. elicit the present equivalent $X_{p}$ of a temporal prospect that pays $X_{0}$ today and $X_{k}$ at date $k$ :

$$
v\left(X_{p}\right)=v\left(X_{0}\right)+D(k) \cdot v\left(X_{k}\right)
$$

where $\left(X_{0}, X_{k}\right)$ vary but $k$ is fixed so it is only necessary to identify a single point on the discount function.

While (8) and (9) appear to share a common structure, there is an important difference that should be noted. For risk, the certainty equivalent pays equal amounts in both states, and corresponds to where an indifference curve intersects the diagonal in state-payoff space. By contrast for time, the present equivalent pays solely on a single date, and corresponds to where an indifference curve intersects the axis in time-dated payoff space. Thus the procedures used to measure utility for risk and time are not completely comparable. ${ }^{18}$

\footnotetext{
${ }^{17}$ This discussion restricts attention to their analysis of the gain domain.

${ }^{18}$ Recall that the impact of diminishing marginal utility upon the slope of an indifference curve is maximized at the axis, and vanishes at the diagonal, so this distinction is not inconsequential.
} 
Abdellaoui et al. (2013) elicit $X_{c}$ and $X_{p}$ using an iterative procedure to find the point of indifference. This is theoretically not incentive compatible if a subject recognizes how her response to one item influences the choices offered to her subsequently. In their first experiment all choices were hypothetical, so this objection is moot; in a second experiment each subject had one chance in twenty that one choice would count for payment. In this second experiment there were 52 French student subjects, with parameters $p=0.25, k=6$ months, seven payoff combinations each for risk and time, and payoffs ranging up to EUR 200.

Abdellaoui et al. (2013) estimate a CARA utility function which is not directly comparable to the CRRA specification reported elsewhere. They find that utility for risk is significantly concave, whereas for time the null hypothesis of linear utility cannot be rejected. The estimated utility curvature parameters for risk and time differ significantly from one another.

To compare utility for risk and time without interacting the domains, Cheung (2015b) transposes the logic of the Holt and Laury (2002) risk preference task into time-dated payoffs. In Holt and Laury, the safe lottery offers more equal payoffs across good and bad states, whereas payoffs in the risky lottery are more unequal. Risk aversion is identified as a preference for the safe lottery even at high probabilities of the good state.

To extend this logic to choice over time, Cheung constructs choice lists over vectors of payments on two dates. One option is smaller sooner, in that it pays less in total but more on the sooner date, while the other is larger later. Interest rates are varied by adjusting the back-end delay, holding the payoffs fixed. ${ }^{19}$ In some choice lists, the smaller sooner option is more evenly distributed over payment dates than the larger later one, while in others this relation is reversed. In this manner - by varying interest rates orthogonally to implications for intertemporal substitution - discounting and utility curvature are separately identified.

By construction of the payoffs, a subject with linear utility is predicted to make the same choices in every list. However, a subject with concave utility will favor a more evenly spread payoff vector - both when it is smaller sooner and when it is larger later. This subject thus chooses the smaller sooner option at higher interest rates in choice lists where it is more evenly spread, compared to where it is not.

Cheung (2015b) conducts an experiment using 122 Australian student subjects, with $t=1$ week, $k$ declining from 27 to 0 weeks (generating interest rates that increase from $25 \%$ to infinity), ${ }^{20}$ and payoffs up to AUD 38. Subjects also completed a single Holt and Laury risk choice list using matched payoffs.

As implied by concave utility, Cheung finds that subjects prefer more evenly spread payoffs, choosing smaller sooner more when it is the more evenly distributed option in the choice list. This difference is highly significant, with $p<0.001$ in a signed-ranks test. Yet its size is modest: on average 0.5 rows per list of ten choices; under risk, the same subjects make 2.1 more safe choices than predicted by linear utility. The estimated instantaneous utility function is significantly concave - in contrast to Abdellaoui et al. (2013) - but less concave than Bernoulli utility elicited under risk, with a CRRA coefficient of 0.02 for time compared

\footnotetext{
${ }^{19}$ This is analogous to varying the probability of the good state over the rows of the choice list in Holt and Laury.

${ }^{20}$ Continuing the analogy to Holt and Laury, the final decision in each list involved a dominated alternative.
} 
to 0.55 for risk (under expected utility). As a result, the estimated annual discount rate falls from $63.9 \%$ assuming linear utility to $62.6 \%$ adjusting for the curvature of instantaneous utility, ${ }^{21}$ and the confidence intervals largely overlap. Thus while instantaneous utility is indeed concave, the effect of correcting for it is much smaller than in the joint elicitation approach where utility is inferred from choice under risk.

\section{Eliciting discounting without utility}

The approaches reviewed in Section 5.3 infer utility directly from choices over time, and compare utilities estimated separately for risk and time. These methods do not presuppose any equivalence between utility concepts, but involve the measurement of both utility and discounting. Analogous to the probability-based methods reviewed in Section 4.2, it may be attractive to consider the possibility of bypassing utility to identify the discount function directly - only without presuming equivalence of utility for risk and time.

A recent paper by Attema et al. (2015) attempts this by moving beyond the framework of equation (3) in which choices involve at most two payment dates - to elicit preferences over quasi-continuous streams of payments. The approach is analogous to the probability-based design of Laury et al. (2012) in that it involves a single nonzero payoff $M$, allowing its utility $v(M)$ to cancel out of any indifference relation. However instead of making choices over the probability with which $M$ is paid, subjects make choices over the subset of dates on which they receive payment, from some overall horizon $T$. Since no risk is involved, in contrast to Laury et al. (2012), it is not necessary to assume equivalence of utility for risk and time.

For example, if a subject is indifferent between receiving $M$ on every date from 1 to $\tau$ (and nothing on any other date) or the same amount $M$ on every date from $\tau+1$ to $T$, one can infer that:

$$
\sum_{t=1}^{\tau} D(t) \cdot v(M)=\sum_{t=\tau+1}^{T} D(t) \cdot v(M)
$$

where $v(M)$ cancels out on both sides. In this manner it is possible to construct inferences about integrals of the discount function from which, given sufficient data, the underlying function may be recovered.

To infer information about the discount function from any of the designs reviewed in this survey, it is necessary to assume that subjects will consume payment on the date that it is received. However, this requirement is particularly strong when there are multiple payment dates: for $v(M)$ to drop out of (10), one must assume that every payment will be consumed at the time that it is received.

Attema et al. (2015) conduct an experiment using 104 Dutch student subjects, with parameters $M=$ EUR 20 and $T=52$ weeks. One subject in each session received real payment for one of her choices.

\footnotetext{
${ }^{21}$ The estimation procedure to correct for non-linear utility is essentially identical to the joint elicitation framework of Andersen et al. $(2008,2014)$, differing only in the source of information used to identify the curvature of utility.
} 


\begin{tabular}{|c|c|c|c|}
\hline & \multicolumn{2}{|c|}{ Assume equivalence? } \\
\hline & & Yes & No \\
\hline \multirow{4}{*}{ Measure utility? } & \multirow{3}{*}{ Yes } & Section 4.1 & Section 5 \\
\hline & & Andersen et al. (2008) & $\begin{array}{c}\text { Andreoni and Sprenger (2012a) } \\
\text { Abdellaoui et al. (2013) } \\
\text { Cheung (2015b) }\end{array}$ \\
\hline & & Section 4.2 & Section 6 \\
\hline & No & $\begin{array}{l}\text { Laury et al. (2012) } \\
\text { Takeuchi (2011) }\end{array}$ & Attema et al. (2015) \\
\hline
\end{tabular}

Table 1: Summary of time preference elicitation strategies

Attema et al. find that the standard exponential discount function fits best among the alternatives they consider. They estimate an annual discount rate of 35\%, which does not differ significantly from that estimated using a version of joint elicitation. This is an intriguing result: since joint elicitation infers utility from risky choices, while Attema et al.'s method makes no assumption about utility, it indirectly suggests that utility may not differ for risk and time. This is in contrast to Abdellaoui et al. (2013) and Cheung (2015b), who measure utility separately for risk and time and find them to differ.

\section{Discussion}

Table 1 classifies the studies reviewed in this survey on two dimensions: whether utility under risk is assumed to be the same as utility over time, and whether the procedure yields a measure of utility. Looking firstly at designs that involve a measurement of utility (the top row of Table 1), joint elicitation (Section 4.1) infers Bernoulli utility $u(\cdot)$ from a risk preference task, whereby utility is found to be substantially concave. By contrast the studies reviewed in Section 5 infer instantaneous utility $v(\cdot)$ directly from choices over time, finding that it is only mildly concave and in fact quite close to linear. From this, it would be tempting to conclude that the former approach should be avoided, and the latter preferred.

However, before jumping to this conclusion, recall that joint elicitation embeds within it the classic binary choice design that assumes linear utility - it is only at the estimation stage that risk and time data are combined and the risk-elicited utility function superimposed. It is thus a strength of joint elicitation that it allows for the discount function to be estimated both assuming equivalence of utility for risk and time and under linear utility. For this reason, the approach has some inherent robustness to the possibility that instantaneous utility may be near-linear. Moreover in many applied and field settings, subjects' risk preferences are of independent interest, so it is useful to collect data on both risk and time preferences irrespective of whether they are combined at the estimation stage. On the other hand, the probability-based methods reviewed in Section 4.2 are indeed best avoided, given that they necessarily presuppose equivalence of utility for risk and time, and moreover - by design - they do not yield any measure of the implied utility function. 
The research reviewed in this survey is very recent, so it is difficult to make definitive recommendations beyond what has been argued above. However there is a clear need for further research along the lines of the studies reviewed in Section 5.3, which compare utilities elicited separately for risk and time. In particular, the finding of near-linear utility over time should be verified and better understood. Only by making explicit measurements - and not bypassing utility altogether, however appealing this may be - will it be possible to empirically adjudicate the validity of competing designs. Despite this current state of uncertainty, the developments reviewed in this survey have indeed brought significant advances in understanding - and the field of time preference remains a rich and exciting one for experimental research.

\section{References}

Abdellaoui, M., Bleichrodt, H., l'Haridon, O., Paraschiv, C., 2013. Is there one unifying concept of utility? An experimental comparison of utility under risk and utility over time. Management Science 59 (9), 2153-2169.

Andersen, S., Harrison, G. W., Lau, M. I., Rutström, E. E., 2008. Eliciting risk and time preferences. Econometrica $76(3), 583-618$.

Andersen, S., Harrison, G. W., Lau, M. I., Rutström, E. E., 2011. Multiattribute utility theory, intertemporal utility and correlation aversion. Working paper 2011-04, Center for the Economic Analysis of Risk, Georgia State University.

Andersen, S., Harrison, G. W., Lau, M. I., Rutström, E. E., 2014. Discounting behavior: A reconsideration. European Economic Review 71, 15-33.

Andreoni, J., Kuhn, M. A., Sprenger, C., 2015. Measuring time preferences: A comparison of experimental methods. Journal of Economic Behavior and Organization 116, 451-464.

Andreoni, J., Sprenger, C., 2012a. Estimating time preferences from convex budgets. American Economic Review 102 (7), 3333-3356.

Andreoni, J., Sprenger, C., 2012b. Risk preferences are not time preferences. American Economic Review 102 (7), 3357-3376.

Attema, A. E., Bleichrodt, H., Gao, Y., Huang, Z., Wakker, P. P., 2015. Measuring discounting without measuring utility. Working Paper, Erasmus University Rotterdam.

Cheung, S. L., 2015a. Comment on "Risk preferences are not time preferences": On the elicitation of time preference under conditions of risk. American Economic Review 105 (7), 2242-2260.

Cheung, S. L., 2015b. Eliciting utility curvature and time preference. Working Paper 2015-01, School of Economics, The University of Sydney.

Coller, M., Williams, M. B., 1999. Eliciting individual discount rates. Experimental Economics 2 (2), $107-127$.

Ebert, S., van de Kuilen, G., 2015. Measuring multivariate risk preferences. Working Paper, Tilburg University.

Epper, T., Fehr-Duda, H., 2012. The missing link: Unifying risk taking and time discounting. Working paper 096, Department of Economics, University of Zurich. 
Epper, T., Fehr-Duda, H., 2015. Comment on "Risk preferences are not time preferences": Balancing on a budget line. American Economic Review 105 (7), 2261-2271.

Epstein, L. G., Tanny, S. M., 1980. Increasing generalized correlation: A definition and some economic consequences. Canadian Journal of Economics 13 (1), 16-34.

Epstein, L. G., Zin, S. E., 1989. Substitution, risk aversion, and the temporal behavior of consumption and asset returns: A theoretical framework. Econometrica 57 (4), 937-969.

Fehr-Duda, H., Epper, T., 2012. Probability and risk: Foundations and economic implications of probability-dependent risk preferences. Annual Review of Economics 4 (1), 567-593.

Frederick, S., Loewenstein, G., O’Donoghue, T., 2002. Time discounting and time preference: A critical review. Journal of Economic Literature 40 (2), 351-401.

Halevy, Y., 2008. Strotz meets Allais: Diminishing impatience and the certainty effect. American Economic Review 98 (3), 1145-62.

Harrison, G. W., Lau, M. I., Rutström, E. E., 2013. Identifying time preferences with experiments: Comment. Working paper 2013-09, Center for the Economic Analysis of Risk, Georgia State University.

Harrison, G. W., Lau, M. I., Williams, M. B., 2002. Estimating individual discount rates in Denmark: A field experiment. American Economic Review 92 (5), 1606-1617.

Harrison, G. W., Rutström, E. E., 2008. Risk aversion in the laboratory. Research in Experimental Economics 12, 41-196.

Holt, C. A., Laury, S. K., 2002. Risk aversion and incentive effects. American Economic Review 92 (5), 1644-1655.

Laury, S. K., McInnes, M. M., Swarthout, J. T., 2012. Avoiding the curves: Direct elicitation of time preferences. Journal of Risk and Uncertainty 44 (3), 181-217.

Quiggin, J., 1982. A theory of anticipated utility. Journal of Economic Behavior and Organization 3 (4), 323-343.

Richard, S. F., 1975. Multivariate risk aversion, utility independence and separable utility functions. Management Science 22 (1), 12-21.

Schmidt, U., 2014. Risk preferences may be time preferences: A comment on Andreoni and Sprenger (2012). Working paper 1942, Kiel Institute for the World Economy.

Takeuchi, K., 2011. Non-parametric test of time consistency: Present bias and future bias. Games and Economic Behavior 71 (2), 456-478. 